\title{
Design Advance Database Supported with some of Data Mining Ensemble for Early Deduction of Osteoporosis
}

\author{
Anhar Khairy Al-Deen \\ Dept. Of MIS \\ College Of Economics \& Business \\ Administration \\ University of Mosul, Nineveh, \\ IRAQ
}

\author{
Kubais Saeed Fahady \\ Ajman University Of Science \& \\ Technology-UAE
}

\author{
Reem Ali AL-Jarah \\ Density college \\ University of Mosul, Nineveh,
}

IRAQ

\begin{abstract}
A computerize Osteoporosis system has been designed, it is based on a database with factors that represent the cause of Osteoporosis, in this database the Visual Basic.net language were used for designing system forms , as well as TSQL language and SQL Server.

Data Mining is considered to be the most important tool used to extract information from data and uncover its hidden paten. The Utility of its tools enable researchers to use it in many applications and to data irrelative of its size.

In this paper the Naïve Bayes Classifier and Rule Induction, were used as a data mining tools, to classify person with, or without, Osteoporosis, and to detect the main causes that leads to Osteoporosis.

Data was collected from a sample of patient, from Al-Salam and Al- Jamhori Hospitals in Mosul City. Direct interview to those patient were used to collect information about the history and the stage of the cases. Based on this data a Model for the Osteoporosis Stages was derived and software was designed, to be used by specialist in the diagnosis and treatment of the disease. The study reached some conclusion and suggestion
\end{abstract}

\section{General Terms}

Osteoporosis ,Database, Protocol ,Classification

\section{Keywords}

Risk Factors, BMD, ADO,ODBC, HTTP , FTP

Rule Induction, Naïve Bayes.

\section{INTRODUCTION}

Data mining could be defined as: Data processing using sophisticated data search capabilities and statistical algorithms to discover patterns and correlations in large preexisting databases[1].

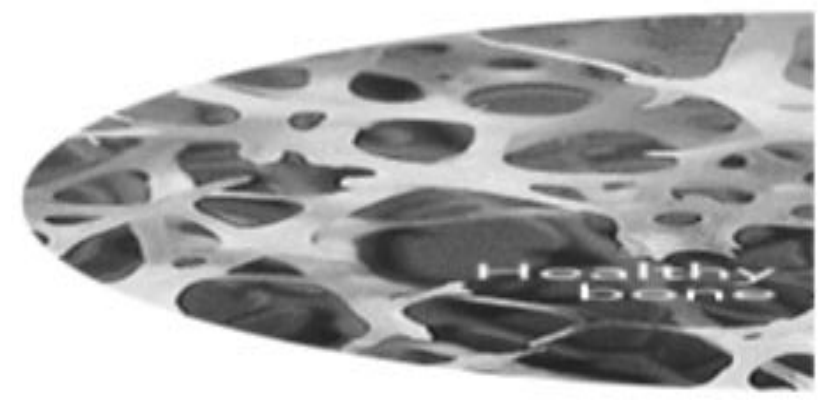

The accessibility and abundance of data make Knowledge Discovery and Data Mining a matter of considerable importance and necessity. Given the recent development in this field, it is not surprising that a wide variety of methods are available to the researchers and practitioners; Rule induction and Naïve Bayes Classifier are some of these methods are available to the researchers and practitioners; Rule induction and Naïve Bayes Classifier are some of these methods.

The aims of this paper are:

- Designs the Osteoporosis data base system that enable specialist discover the diseases at early stages; and to be used by researchers to improve the knowledge about the disease.

- Introduce procedures that may be used to trace the causes lead to Osteoporosis and to select the most reliable one.

- Using the Naïve Bayes Classifier to classify the patient with or without Osteoporosis with relatively small Bayes error rate.

Consequently, the work will be divided in two main parts:

1. The theoretical part: in which the concept of Osteoporosis, Data Base facilities , Web application servers ,Data Mining Tools (Rule Induction, Naïve Bayes Classifier), will be introduced.

2. The empirical part: in which the source of data, collection, classifying, and analyzing will be reviewed. Practical steps for both Rule Induction and Naïve Bayes Classifier will be discussed. And an Osteoporosis data base system with some protocols, like (HTTP, FTP ), will be developed.

The findings in both theoretical and empirical parts shall be discussed and final conclusions will be listed.

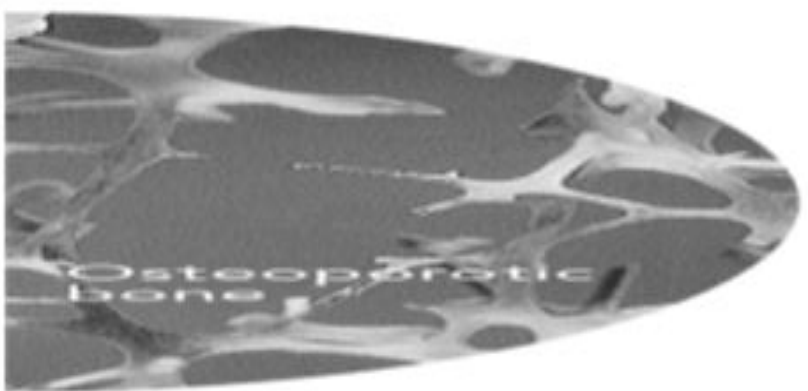

Fig 1:compare the form of healthy\& Osteoporotic bone 


\section{WHAT IS OSTEOPOROSIS}

Osteoporosis is a condition characterized by a decrease in the density of bone, decreasing its strength and resulting in fragile bones. Osteoporosis literally leads to abnormally porous bone that is compressible, like a sponge.

This disorder of the skeleton weakens the bone and results in (breaks) in the bones. Normal bones composed of protein, collagen, and calcium, all of which give bone its strength. Bones that are affected by osteoporosis can fracture with relatively minor injury that normally would not cause a bone to fracture. The Fracture can be either in the form of cracking or collapsing[2]

\subsection{Risk Factors of Osteoporosis}

The risk factors for osteoporosis are numerous, many are inter-correlated and the relevance of risk factors differs between individuals.

There are varieties of factors that represent a Risk factor to have Osteoporosis; the National Osteoporosis Foundation in USA has classified the Risk factors as controllable and uncontrollable[3]

\section{Uncontrollable Risk Factors}

- $\quad$ Being over age 50.

- Being female.

- Menopause.

- Family history of osteoporosis.

- Low body weight/being small and thin.

- Broken bones or height loss.

\section{Controllable Risk Factors}

- Not getting enough calcium and vitamin D.

- Not eating enough fruits and vegetables.

- Getting too much protein, sodium and caffeine.

- Having an inactive lifestyle.

- Smoking.

- Drinking too much alcohol.

- Losing weight.

The following are other factors that may increase the risk of developing osteoporosis

- Malabsorption (nutrients are not properly absorbed from the gastrointestinal system) from conditions .

- Low estrogen levels in women (such as that occurs in menopause or with early surgical removal of both ovaries).

- Low testosterone levels in men (hypogonadism).

- Chemotherapy that can cause early menopause due to its toxic effects on the ovaries

- Chronic inflammation, due to chronic diseases such as rheumatoid arthritis or liver diseases

- Hyperthyroidism, a condition wherein too much thyroid hormone is produced by the thyroid gland (as in Grave's disease) or is ingested as thyroid hormone medication

- Lack of vitamin D, when the body cannot absorb adequate amounts of calcium from the diet to prevent osteoporosis.

\subsection{Bone density measure}

Different measures are used to determine the bone health, the most commonly used is the Bone Mineral Density test (BMD)The test can identify osteoporosis, and determine the risk for fractures, and measure the response to osteoporosis treatment. The most widely recognized BMD test is called a dual-energy x-ray absorptiometry, or DXA test. The test can measure bone density at the hip and spine[4]. The DXA test results are usually compared to the ideal (or peak) bone mineral density of a healthy 30 -year-old adult to determine a T-score. By the value of the BMD-SDT_SCORE the patient is classified as follows [5][6]:

If :

$$
\begin{array}{llll}
\text { BMD }>-1 \quad \text { DT SCORE } & \rightarrow & \text { Normal } \\
-2.5<\text { BMD }<-1 \quad \text { DT SCORE } & \rightarrow & \text { Osteopenia } \\
\text { BMD }<-2.5 D T \quad \text { SCORE } & \rightarrow & \text { Osteoprosis }
\end{array}
$$

Where the Osteopenia is consider to be a precursor to osteoporosis.

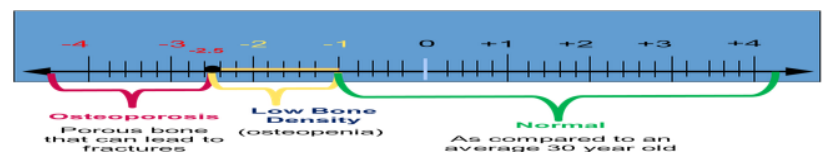

\section{DATA BASE FACILITIES 3.1 Active Data Object}

VB.NET includes a number of new controls for accessing data from your application.

Applications communicate with a database, firstly, to retrieve the data stored there and present it in a user-friendly way, and secondly, to update the database by inserting, modifying and deleting data.

ADO.NET is a data access technology from Microsoft .Net Framework, which provides communication between relational and non-relational systems through a common set of components. ADO.NET consist of a set of Objects that expose data access services to the .NET environment. ADO.NET is built for disconnected architecture, so it enables truly disconnected Data Access and Data Manipulation through its Dataset Object, which is completely independent from the Data Source[7].

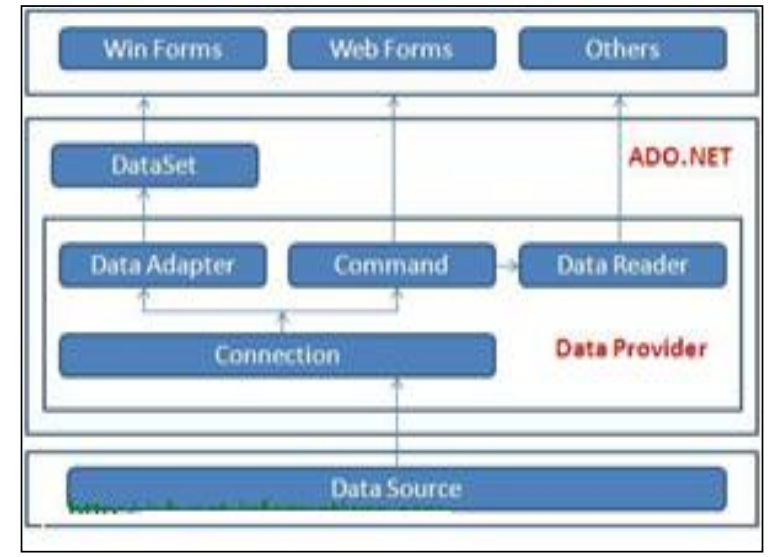

Fig 2:ADO.NET

The structured process flow through various components. The object model can be pictorially described as: 


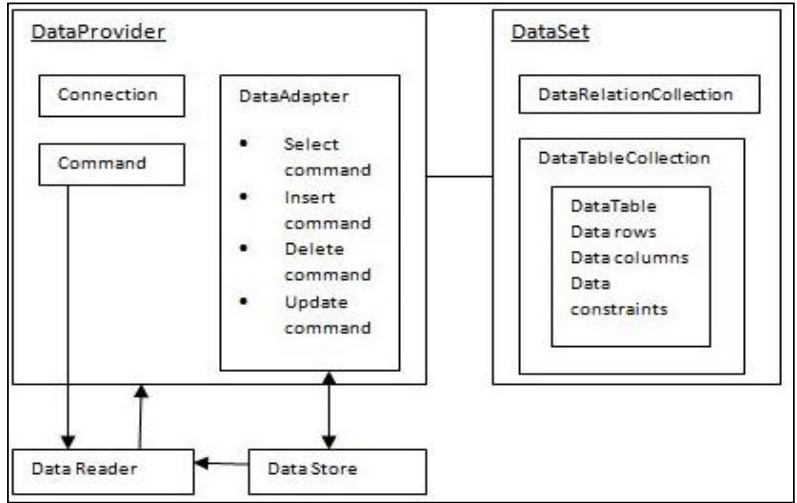

Fig 3: Object model

There are following different types of data providers included in ADO.Net

- The .Net Framework data provider for SQL Server provides access to Microsoft SQL Server.

- The .Net Framework data provider for OLE DB provides access to data sources exposed by using OLE DB.

- The .Net Framework data provider for ODBC - provides access to data sources exposed by ODBC

Open Database Connectivity (ODBC) provides a set of application programming interface (API) functions which make it easier for a developer to connect to a wide range of database formats[8].

ODBC drivers are DLLs(Dynamic Link Library) that contain the functions that let you connect to various databases. Each ODBC driver is separate for each database format. ODBC drivers take the code from a program and convert the functions to the specific database format being used. Connections-shown in figure 4

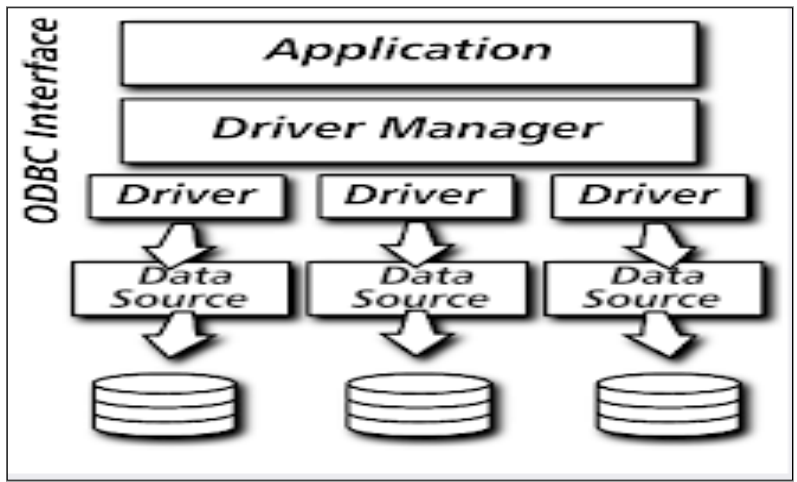

Fig 4: Architecture ODBC

Advanced Query Tool uses ODBC drivers to connect to database. Before you can use AQT with your database, you will need to install and configure both the ODBC Driver and the Database Client. AQT is reliant on the ODBC drivers to operate correctly; many of the limitations and bugs you see in AQT are because of limitations and bugs in the ODBC drivers.

The following diagram gives a high-level view of the interaction between AQT, the ODBC Driver and the Database Client. Note that for some databases the functionality of the Database Client is part of the ODBC Driver.

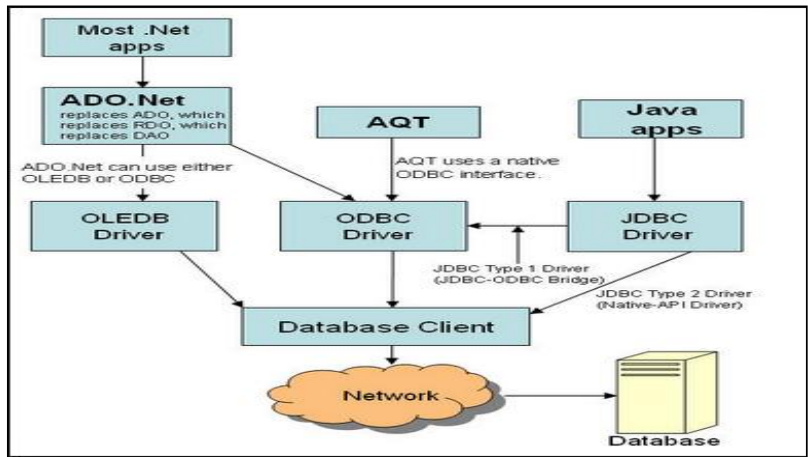

Fig 5: interaction between AQT, the ODBC Driver and the Database

\section{WEB APPLICATION SERVERS}

Web application servers can be used to:

Connect to and query a database from a Web page.

Present database data in a Web page, using various formats.

Create dynamic Web search pages.

Create Web pages to insert, update, and delete database data.

Enforce referential integrity in the application program logic.

Use simple and nested queries and programming logic to represent business rules.

Web application servers provide features such as:

- An integrated development environment with session management and support for persistent application variables.

- Security and authentication of users through user IDs and passwords.

- Computational languages to represent and store business logic in the application server.

- Automatic generation of HTML pages integrated with Java, JavaScript, VBScript, ASP, and so on.

- Performance and fault-tolerant features.

- Database access with transaction management capabilities.

- Access to multiple services, such as file transfers (FTP), database connectivity, e-mail, and directory services.

- Network Component provides an easy-to-use development interface to a variety of IP protocols. By using Network Component, you can very easily create or enhance applications with network features.

Network Component features the following: DNS, FTP, HTTP, HTTPs, ICMP Ping, IP-to-Country, MSN, NTP, RSH, SCP, SFTP, SNMP v1/v2c (Get, GetNext, Set), SNMP Traps, SNMP MIB, SSH, TCP, Telnet, TFTP, UDP, Telnet, WakeOn-LAN and more.

Network Component can be well integrated into any development platform that supports ActiveX objects.

\subsection{Web Transfer control}

The most common networking control supported by web transfer control is "File Transfer Control" (FTP), \& "Hypertext Transfer Protocol" (HTTP).

A protocol is asset of rules that governs data communication. A protocol defines what is communicated, how it 
communicated, and when it is communicated [9] .

\subsubsection{File Transfer Protocol (FTP)}

Stands for "File Transfer Protocol." FTP or transferring files FTP is a standard internet protocol for transmitting files between computers on the Internet [10].

To transfer files with FTP, you use a program often called the client. An FTP client program initiates a connection to a remote computer running FTP server software.

After the connection is established, the client can choose to send and/or receive copies of files, singly or in groups. To connect to an FTP server, a client requires a username and password[11] as set by the administrator of the server. Many public FTP archives follow a special convention for that accepts a username of "anonymous."[12].

Using File Transfer Protocol (FTP):

FTP uses a TCP control and data connection. The TCP control connection is used for opening / closing an FTP session and for transferring commands from client to server. The data connection is used for transferring individual files between client and server. Every file transfer uses a separate data connection.[13]

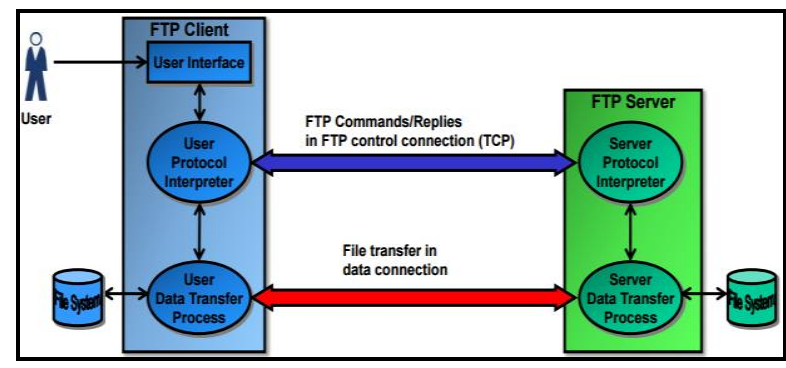

Fig 6 :File Transfer Protocol

FTP can transfer file types: A SCII,IMAGE, EBCDIC mode across the data connection [14]

\subsubsection{Hypertext Transfer Protocol (HTTP)}

HTTP - the Hypertext Transfer Protocol - provides a standard for Web browsers and servers to communicate. The definition of HTTP is a technical specification of a network protocol that software must implement.

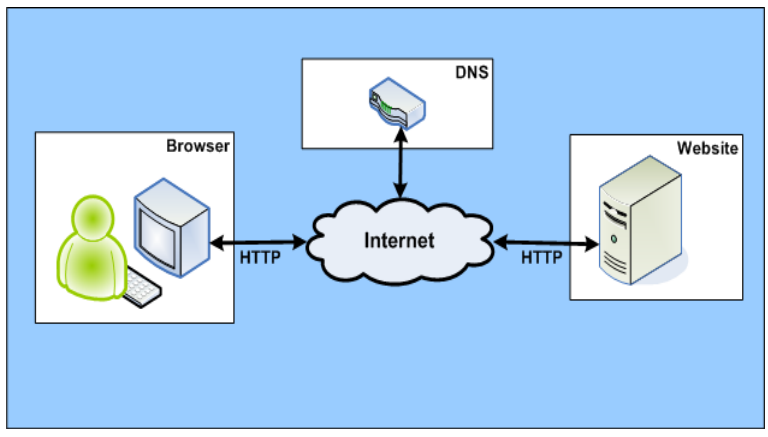

Fig 7:Interaction between Browser \& Web Server

HTTP is an application layer network protocol built on top of TCP. HTTP clients (such as Web browsers) and servers communicate via HTTP request and response messages. The three main HTTP message types are GET, POST, and HEAD [15] .

Every client and server on the Web is identified by a unique numerical IP address or Uniform Resource Locator (URL). This ensures that requests for data and corresponding responses (datagrams) get routed correctly through the various networks that collectively make up the World Wide Web. Numerical IPs are mapped to an associated name to make the URL easier to remember

Practical information systems require more functionality than simple retrieval, including search, front-end update, and annotation. HTTP allows an open-ended set of methods and headers that indicate the purpose of a request [16].

\section{DATA MINING ENSEMBLE 5.1 Rule Induction}

In the classification task of data mining, one way of representing the knowledge discovered by the data mining algorithm consists of a set of classification rules, where each rule has the form: IF <conditions> THEN <predicted class>. This knowledge representation has the advantage of being intuitively comprehensible to the user [17].

There are a number of rule induction algorithms can be proposed to discover classification rules[18]

The basic rule induction method is :

For each class C

Initialize to the set of all examples $\mathrm{E}$

While $\mathrm{E}$ contains examples in class $\mathrm{C}$

Create a rule $\mathrm{R}$ with an empty left-hand side that predicts class $\mathrm{C}$

Until $\mathrm{R}$ is $100 \%$ accurate (or there are no more attributes to use) do:

For each attribute A not in $\mathrm{R}$, and each value $\mathrm{V}$

Consider adding the condition (attribute-value pair) $\mathrm{A}=\mathrm{V}$

To the left hand side of $\mathrm{R}$

Select $\mathrm{A}$ and $\mathrm{V}$ to maximize the accuracy and covering of the

Attribute-value pair

Add $\mathrm{A}=\mathrm{V}$ to $\mathrm{R}$

Remove the examples covered by R from B

\subsubsection{Kinds of Rules}

A case ( $x$ ) is covered by a rule (r)"if and only if every situation (attribute value pair) of ( $\mathrm{r}$ ) is satisfied by the corresponding attribute value for $(\mathrm{x})$ ". The $\operatorname{concept}(\mathrm{C})$ defined by the right hand side of rule (r) is indicated by $(r)$. We say that a concept $(\mathrm{C})$ is completely covered by a rule performance set $(\mathrm{R})$ "if and only if for every case (x) from $(C)$ there exists a rule $(r)$ from $(R)$ such that $(r)$ covers $(x) "$.

A rule set $(\mathrm{R})$ is complete "if and only if every concept from the data set is totally covered by $(\mathrm{R})$ ". A rule $(\mathrm{r})$ is consistent (with the data set) if and only if for every case (x) covered by $(\mathrm{r}),(\mathrm{x})$ is a member of the concept $\mathrm{C}$ indicated by $(\mathrm{r})$. A rule set $\mathrm{R}$ is consistent if and only if every rule from ( $\mathrm{R})$ is consistent with the set of data.[19]

Commonly, there are two general types of algorithms for rule induction: top-down and bottom-up algorithms. Top-down approaches 'start from the most general rule and specialize it 
gradually'. Bottom-up methods' star from a basic fact given in training database and generalize it'.[20]

\subsubsection{Comparing of Rule Algorithms}

In the rule induction algorithm literature, comparing different classification algorithms is not a straightforward process. There is a variety of metrics which can be used to estimate how good a classifier is, including classification Accuracy, Sensitivity and Specificity.

Nevertheless, classification Accuracy and Coverage are still the most common metric used to compare classifiers:

The Accuracy is the ratio of the number of correctly classified samples to the total number of samples,[21], The Coverage of an association rule is the ratio of cases in the data that have the attribute values or items specified on the left hand side of the rule.[22] .

\subsection{Naive Bayes classifier}

One of the important classifiers is the naive Bayes method simple Bayes \& independence Bayes, it is also called the "idiot's Bayes".

This classifier is a simple probabilistic classifier based on applying Bayes' theorem (from Bayesian statistics) with strong (naive) independence assumptions. A more descriptive term for the underlying probability model would be "independent feature model".

In spite of their naive design and apparently over-simplified assumptions, naive Bayes classifiers have worked quite well in many complex real-world situations.

This method is important for several reasons, mainly because: It is very easy to construct, does not need complicated iterative parameter estimation schemes which make it applicable to huge data sets, easy to interpret by unskilled users, and particularly important, it often does surprisingly well.

It may not be the best possible classifier in any given application, but it can usually be relied on to be robust and to do quite well.[23] .

As its name suggests, this is a classification method developed from Bayes' theorem. It is a probabilistic classifier based on the Bayes theorem that uses very strong assumptions on the independence between the predictors. The strict property of independence is rarely applicable in the real-world problems, but it may consider as naïve. Nevertheless, this method has been successfully applied to large number of real-world applications. [24] .

It has already been used for calculating the probability $\mathrm{P}\left(\mathrm{Y}_{\mathrm{i}} \mid \mathrm{x}\right)$ of an individual's belonging to a group Yi conditionally on its characteristics $x$ based on the probability $\mathrm{P}\left(\mathrm{X} \mid \mathrm{Y}_{\mathrm{i}}\right)$ of having these characteristics conditionally on its belonging to $\mathrm{Y}_{\mathrm{i}}$. [25]

We also need to know the a priori probability of Yi. According to Bayes' theorem,

\section{$P\left(Y_{1} \mid X\right)-\frac{P\left(X \mid Y_{1}\right) P\left(Y_{1}\right)}{\sum, P\left(X \mid Y_{1}\right) P\left(Y_{1}\right)}$}

Where, the part in the denominator is equal to the probability $\mathrm{P}(\mathrm{x})$. We can reformulate this property in terms normally used for classification problems [26].

This technique remained popular over the years, despite its name (also known as "Idiot's Bayes"). It is especially appropriate when the dimension $\mathrm{p}$ of the feature space is high, making density estimation unattractive[27].

Assuming 'naively' that the variables are independent (conditionally on Y), we can write [26] .

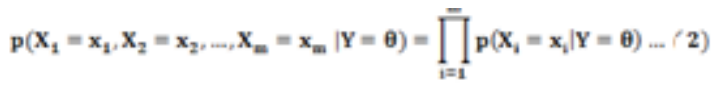

The naive Bayes classification is therefore:

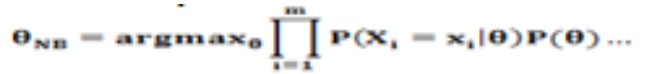

The steps of classifier is show in figure 8

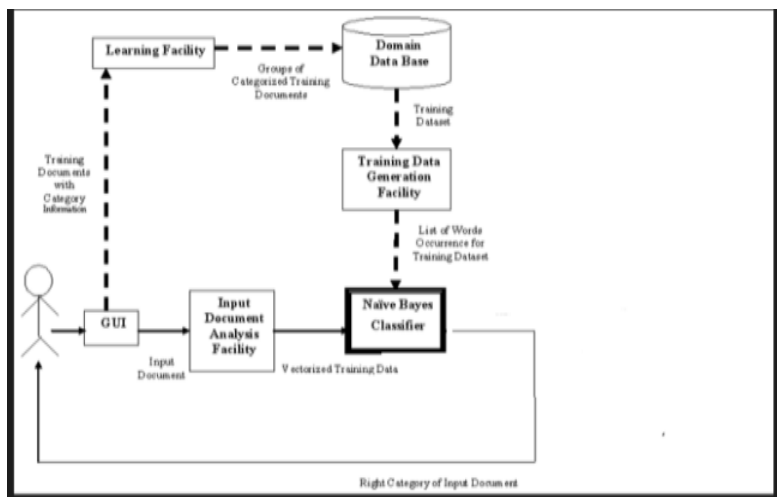

Fig 8: steps of classifier

\subsubsection{Bayes Classifier Error}

The classifier that assigns a vector $\mathrm{x}$ to the class with the highest posterior is called the Bayes classifier. The error associated with this classifier is called the Bayes error, which can be expressed as:

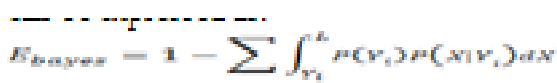

Where $Y_{i}$ is the region where class $i$ has the highest posterior.[28].

One of the simplest bounds for the Bayes error is supplied by the "Mahalanobis distance measure".

For a 2-class problem, let $\Sigma$ be the non-singular, average

One of the simplest bounds for the Bayes error is supplied by the "Mahalanobis distance measure".

For a 2-class problem, let $\Sigma$ be the non-singular, average ovariance matrix, $\Sigma=P\left(Y_{1}\right) \Sigma_{1}+P\left(Y_{2}\right) \Sigma_{2}$, and $\mu_{\mathrm{i}}$

be the mean vector for classes $i=1 ; 2$. Then the Mahalanob is distance $\Delta$, given by[29]:

$\left.\left.\Delta-\omega_{1}-\mu_{2}\right)^{x} \Sigma^{-1} G_{1}-\mu_{2}\right)$

Which provides the following bound on the Bayes Error?

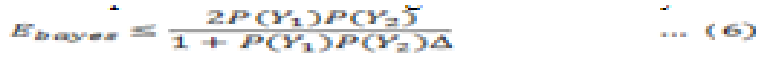

The main advantage of this bound is the lack of restriction on the class distributions. Furthermore, it is easy to calculate using only sample mean and sample covariance matrices. It is therefore, provides a quick way of obtaining an approximation for the Bayes error.

\section{2 .2 Zero-Frequency Cells}

Adjusted probability estimate for zero -Frequency cells by 
using m-estimate method for estimate a conditional probability

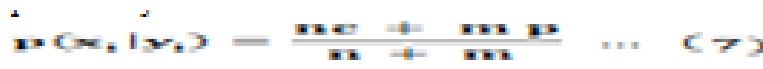

where $\mathrm{n}$ : represents the total number of records for this target class, nc: number of these $\mathrm{n}$ records that also have the attribute value of interest, $\mathrm{p}$ the prior estimate of the probability being estimated, and $\mathrm{m}$ is a constant representing the equivalent sample size [30] .

\subsubsection{Numeric Predictors}

Bayesian classification can be extended from categorical to continuous predictor variables, provided that we know the relevant probability distribution.[31]

$\mathbf{p}\left(\mathbf{X}_{i}=\mathrm{x}_{i} \mid \mathrm{Y}_{\mathrm{i}}=\mathrm{y}_{i}\right)=\frac{1}{\sqrt{2 \pi} \sigma_{i j}} \exp \left[\frac{\left(\mathrm{x}_{i}-\mu_{i j}\right)^{2}}{2 \sigma_{i j}^{2}}\right] \ldots$

\section{Practical Part}

The practical part of this paper was based on a sample of patients calling on two hospitals in Mosul City - Iraq.

The sample included patients with osteoarthritis symptoms who called on the teaching hospitals in ("Al-Jamhuria" and "Al- Salam"). There were 644 cases all of which have been subjected to clinical and radiation examination, 504 of them found to be osteoarthritis patients while the others were not. The following table table(1) shows the classification of these cases according to gender:

\begin{tabular}{|l|l|l|l|}
\hline Diagnosis & Male & Female & Total \\
\hline Osteoarthritis Patient & 194 & 310 & 504 \\
\hline None Osteoarthritis Patient & 80 & 60 & 140 \\
\hline Total & 274 & 370 & 644 \\
\hline
\end{tabular}

\section{.6.1 Information System for Osteoporosis}

\section{- First: The system data analysis}

A description of the organization of a database. It is often created as an entity relationship diagram. Today's modeling tools allow the attributes and tables (fields and records) to be graphically created

Model: The model represents a perception of structures of reality.

Data model: a collection of conceptual tools for describing data, data relationships, data semantics, and consistency constraints. A data model provides a way to describe the design of a database at the physical, logical, and view levels [32].

Or Data modeling translates a specific real-world environment into a data model that represents the real-world data, users, processes, and interactions[33].

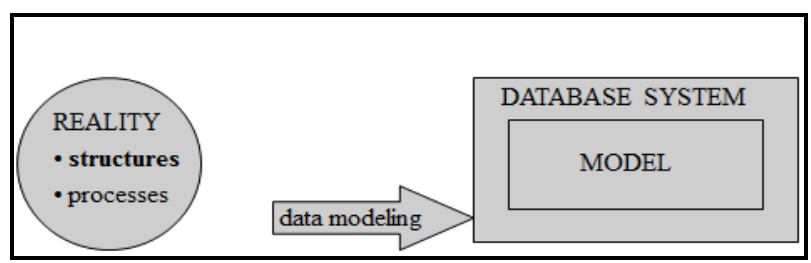

Fig 9:Data Modeling
Data Modeling was used in the analysis of the data system for the following reasons:

1. The need for a level or conceptual model (Conceptual Model): This method provides the basic information at the top level allowing beneficiaries to have an understanding of the high system requirements, providing complete and accurate requirements, and this is one of the most important duty of things offered when designing databases this concept is not supported in the traditional ways.

2. The method of data modeling is interested in the manner in which the data represented in the real world, regardless of the data used in the data storage base type, whether Network or relational or hierarchical and this will allow developers to take correct and complete information to the parts of the system in the real world and only where the focus is on achieving the requirements of the beneficiaries of the system and not stored mechanism.

3. This Method is to clarify and concisely any relational model is to clarify the relationships between tables, fields, and once completed, the conceptual model of relations be clear and the remaining operations to be easy and clear as well.

The style of data modeling (Data Modeling) passes four levels have been applied in the practical side of the research.

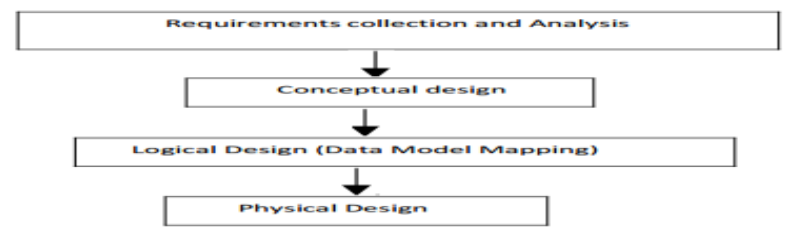

Fig 10:Simplified description of the database design process

(A) The External Level: At this stage, the requirements of the beneficiaries were collected from the system after identifying the people who will deal with the system by conducting interviews with them and figure 11 following shows the beneficiaries of the system and the requirements of each beneficiary.

Requirement of the beneficiaries were collected from the system after identifying people who will deal with the system through interviews with them

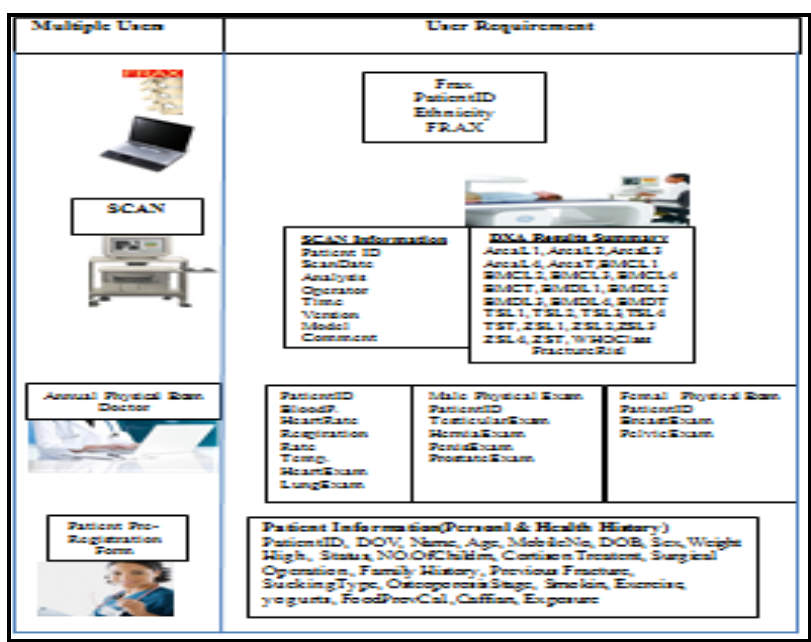

Fig 11 External Level For Osteoporosis System 
(B) Conceptual Data Modeling - identifies the highest-level relationships between different entities.

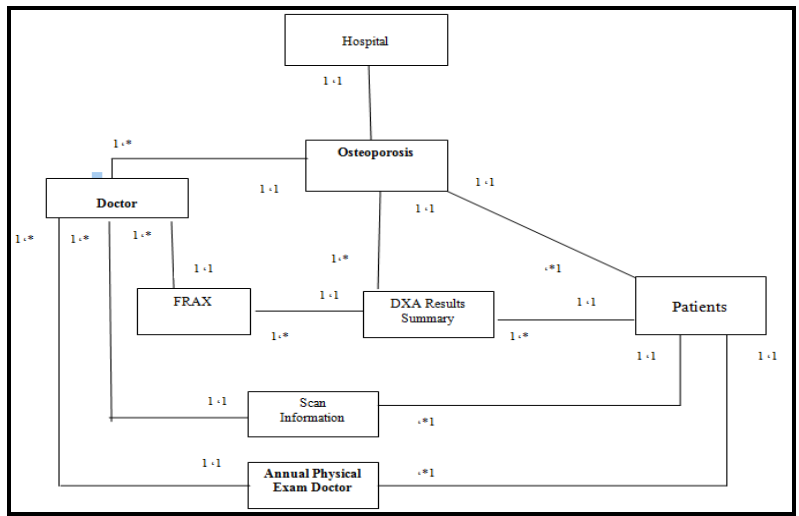

Fig 12:The system's designer entities and the relationships

(C) Logical Data Modeling - illustrates the specific entities, attributes and relationships involved in a business function. Serves as the basis for the creation of the physical data model.

(D) Physical Data Modeling - represents an application and database-specific implementation of a logical data model.

\section{- Second:}

We build an information system for the Osteoporosis cases, By using a (V.B.net), with Active Data Object (ADO) tool and each of HTTP\&FTP protocol for access to our object.

A programming language Transact-SQL (T-SQL) is used, Table are created by this powerful language for performing operations like updating, inserting, and retrieving from/into tables for developing logic reviews. For security, only some granted user can access the stored data. Unauthorized users are banned to retrieve this data.

We can use most Transact-SQL commands; however, some commands (such as CREATE PROCEDURE, CREATE VIEW, SET SHOWPLAN_TEXT, SET SHOWPLAN_ALL, and so forth) must be the first (or only) statement in a command batch..

The main form consists of four menus: Osteoporosis Database, calculation of the fracture risk calculated using the FRAX index, Data mining (Rule induction, Naïve Bayes Classifier), GOTO Figure (13) shows the main form that has been designed.

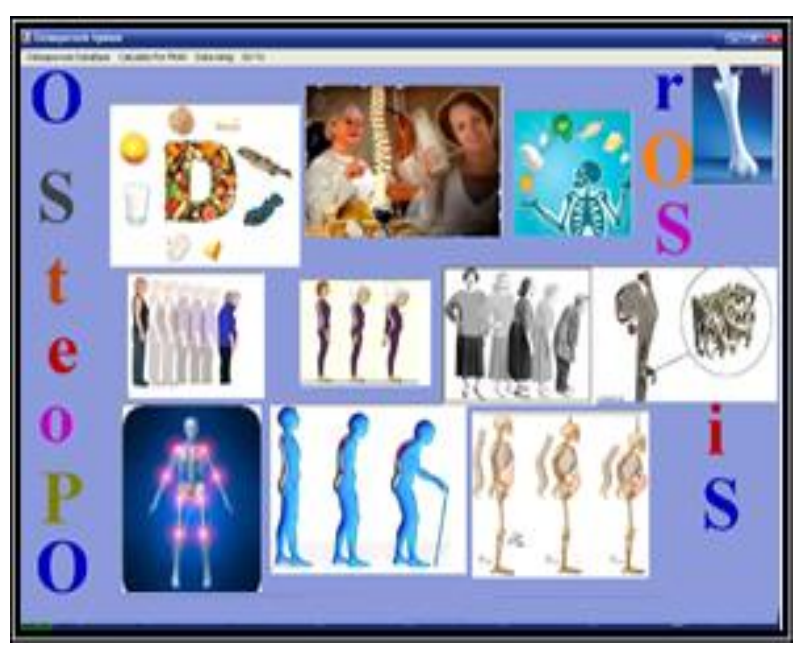

Fig 13: Main Form

\subsubsection{Osteoporosis Database}

There are many form design ,two of them are:

- Patient information(Personal \& Medical History) as show in Figure (14).

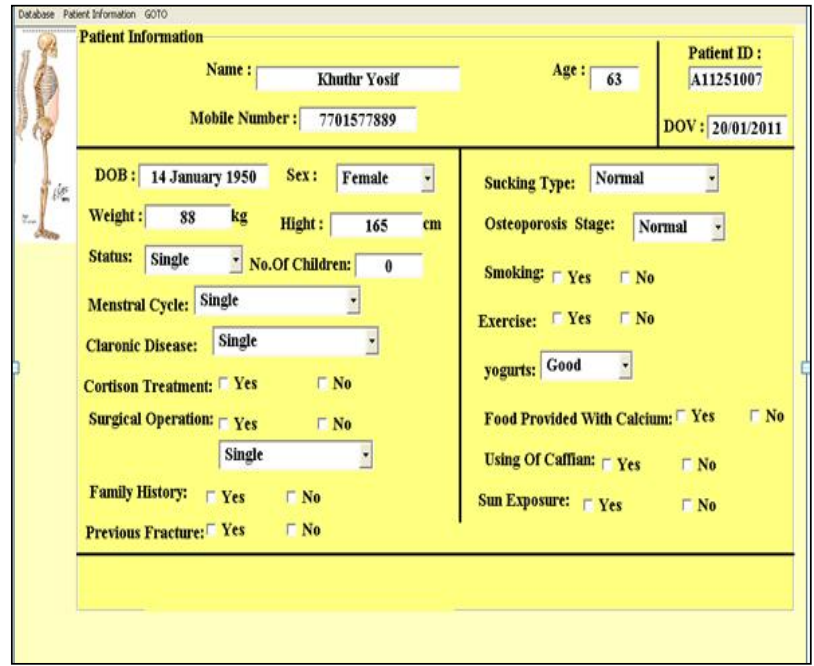

Fig 14: Patient Information Form

- Scan Information

The User go to form Scan Information as shown in Figure(15), in this form the measures of Bone intensity were calculation Total(Area, BMC, BMD, T-Score, Z-score) by using some functions, and after then the (WHO- worth Health Organization), and the Patient Classification have been determined

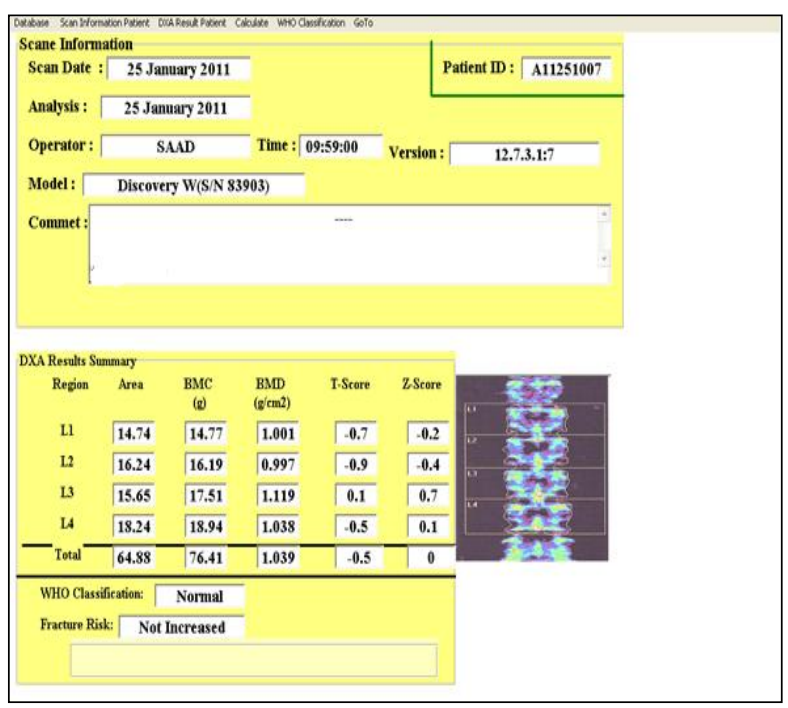

Fig 15:Scan Information Form

Note: Patient with fragility fractures and T-Score above -1.0 should be evaluated for other causes of pathologic fracture, as shown in figure 16 


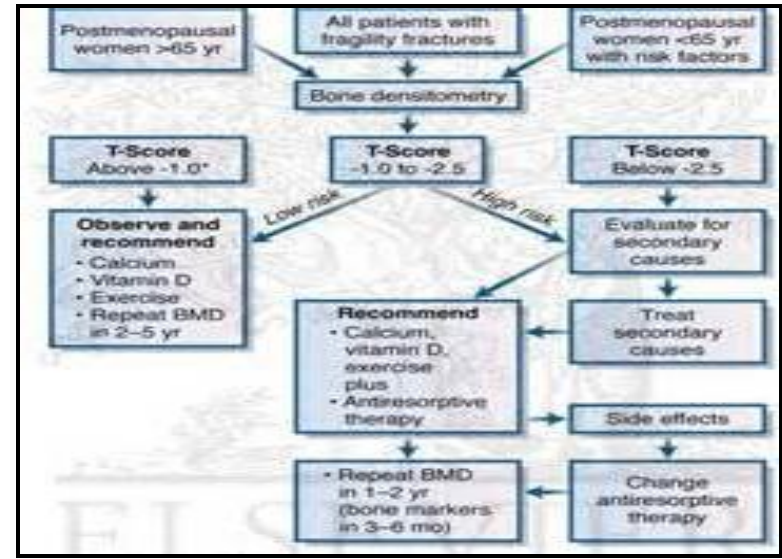

Fig 16: other causes of pathologic fracture

\subsubsection{Calculate For FRAX Menu}

In this part of the system ,both of the connection with net to calculate the Fracture Risk Assessment value for each patient throw the website (http://shef.ac.uk/FRAX/tool.jsp?long=en), and used of FTP Application. This menu contains two commands:

- Website Connection: after click on the command Website Connection the user go to form as shown in Figure(17). First determines if there is a text in combo URL. if so procedure Navigate of web Browser1 was called and passed string in to Combo URL., then web Browser1 display the document associates with a valid URL., it has four stage :

Command Go to display the URL specified by the $U R L$ : user.

Command Back: revisit the previous document in the history list.

Command Forward revisit the Next document in the history list.

Command Home: $\quad$ load the default home page

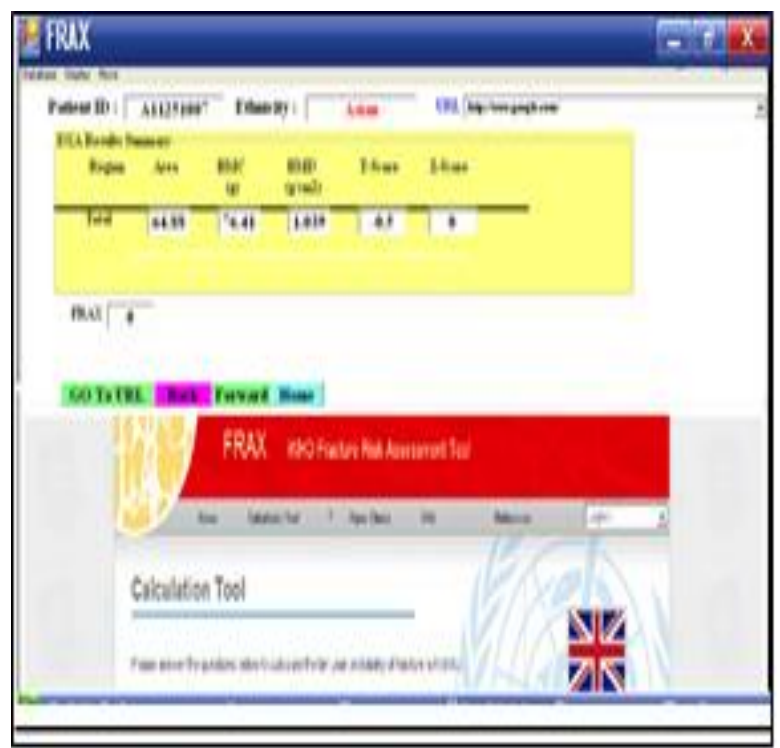

Fig 17: Website Connection Form

Note that : in this form, we recall a total of DXA Result Summary, which we needed as an input data to calculate FRAX from web

The Flowchart of FTP upload is show in figure 18

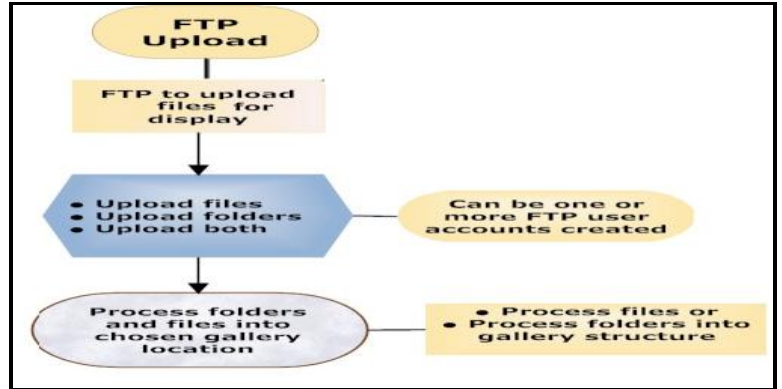

Fig 18:FTP Upload

-Download File: When the program executes the user is presented with the interface as shown in the Figure(19).We provided default values for the FTP site and File to retrieve from Test computer to doctor's computer.

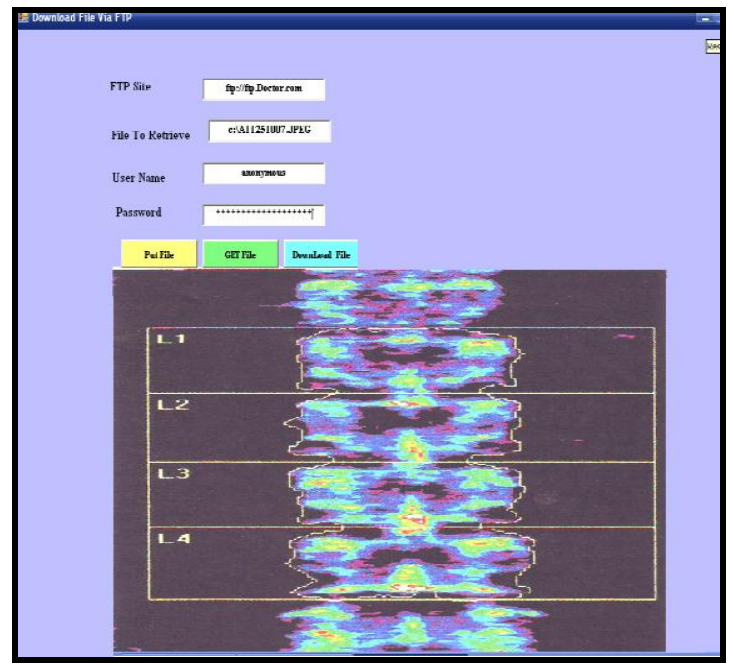

Fig 19: FTP Application to download a file

\subsubsection{Data mining Ensemble}

\section{(A) Osteoporosis Causes Tree}

In this part, we interest to the cause of Osteoporosis, the tree, in the second stage has two leaf nodes, each one split to two other leaf nods, and so on, as show in Figure(20). Each one represents a rule.

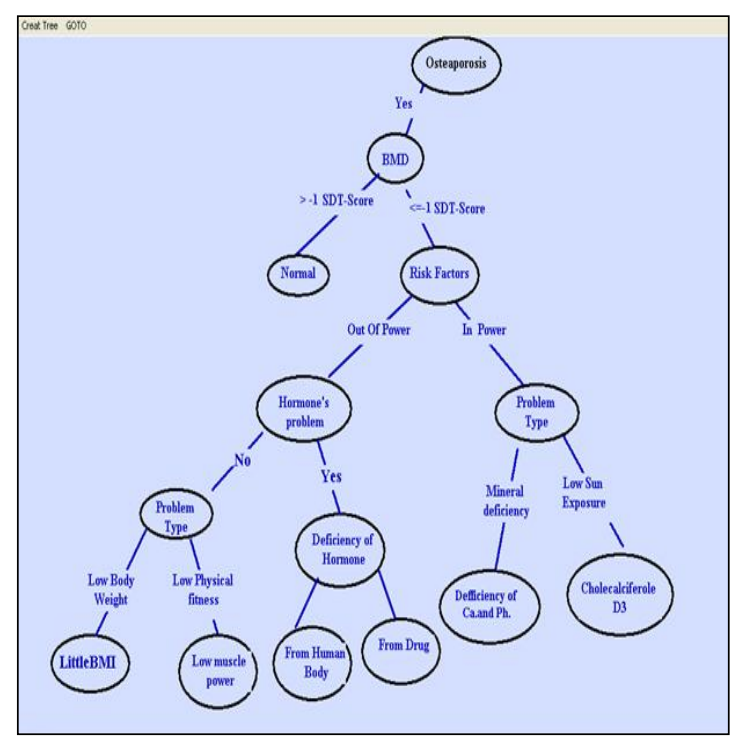

Fig 20:Osteoporosis Causes Tree 
Then, we have the following rules corresponding to the tree:

Rule(1): If the patient has a BMD $\leq-1$ SDT_Score and his risk factor type is (in power) then he suffer from Different Problem.

Rule (2): If the patient has a BMD $\leq-1$ SDT_Score and his risk factor type is (out of power) then he suffer from Hormone Problems.

Rule(3): If the type of patient risk factor is (in power) and his problem is Low Sun Exposure then he has Cholecalciferole D3.

Rule (4): If the type of patient risk factor is (in power) and his problem is Mineral Deficiency then he has a Deficiency of Calcium \& Phosphor.

Rule (5): If the type of patient risk factor is (out of power) and he has a Hormones problem then he has a Deficiency of Hormone.

Rule (6): If the type of patient risk factor is (out of power) and he hasn't had a Hormones problem and his problem is a Low physical Fitness then he has Low muscle power

Rule (7): If the type of patient risk factor is (out of power) and he hasn't had a Hormones problem and his problem is Low Body Weight then he has a Little BMI.

Currently, we want to find a compacted "covering" rule set that completely partitions the patent into their correct cause of the casualty of Osteoporosis. The covering set is found by searching for a single "best" rule that covers cases for only one cause. The value of both of Accuracy and Coverage as below:

\begin{tabular}{|c|c|c|c|c|}
\hline $\begin{array}{l}\text { Rule } \\
\text { (1): }\end{array}$ & Accuracy = & $53 \%$ & Coverage $=$ & $82 \%$ \\
\hline $\begin{array}{l}\text { Rule } \\
\text { (2): }\end{array}$ & Accuracy = & $67 \%$ & Coverage $=$ & $82 \%$ \\
\hline Rule & Accuracy = & $46 \%$ & Coverage $=$ & $53 \%$ \\
\hline
\end{tabular}

(3):

$\begin{array}{llll}\begin{array}{l}\text { Rule } \\ \text { (4): }\end{array} & \text { Accuracy }=54 \% & \text { Coverage }=53 \% \\ \text { Rule } & \text { Accuracy }=41 \% & \text { Coverage }=67 \%\end{array}$

(5):

$\begin{array}{llll}\begin{array}{l}\text { Rule } \\ (6):\end{array} & \text { Accuracy }=59 \% & \text { Coverage }=39 \% \\ \text { Rule } & \text { Accuracy }=41 \% & \text { Coverage }=39 \%\end{array}$
(7):

Note that the rules which have a highly Accuracy (with invariable Coverage) are shade. By comparing of these rules, we conclude that Rule (2),is the best one then Rule(4), Rule (6) respectively. See Appendixes: (1-A) , (1-B)

(C) Naïve Bayes Classifier Tree

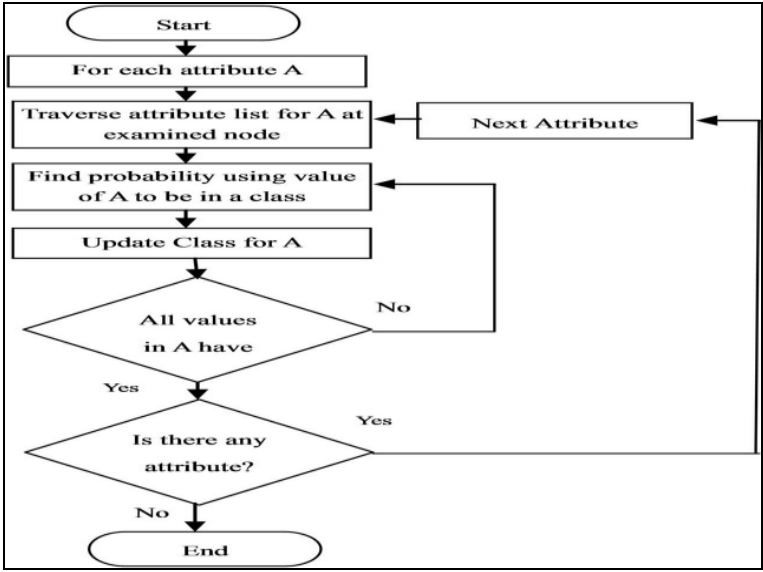

Fig 21: Flowchart of Naïve Bayes decision tree algorithm

To construct the tree of Naïve Bayes classifier for Osteoporosis ,see Appendixes:(2-A),(2-B),(2-C) and (2-D), and for guarantee arrival to helpful classifier, we depend on nine of risk factors which are trust worthy from (WHOworth Health Organization).These factors are:
X1: Age
X6:Parent fractured hip
$\mathrm{X} 2$ : Weight
$\mathrm{X} 7$ :Previous fracture
$\mathrm{X} 3$ : length
X8:Glucocorticoids
$\mathrm{X} 4$ :current menopause
X9:current smoking
X5:Secondary
Osteoporosis

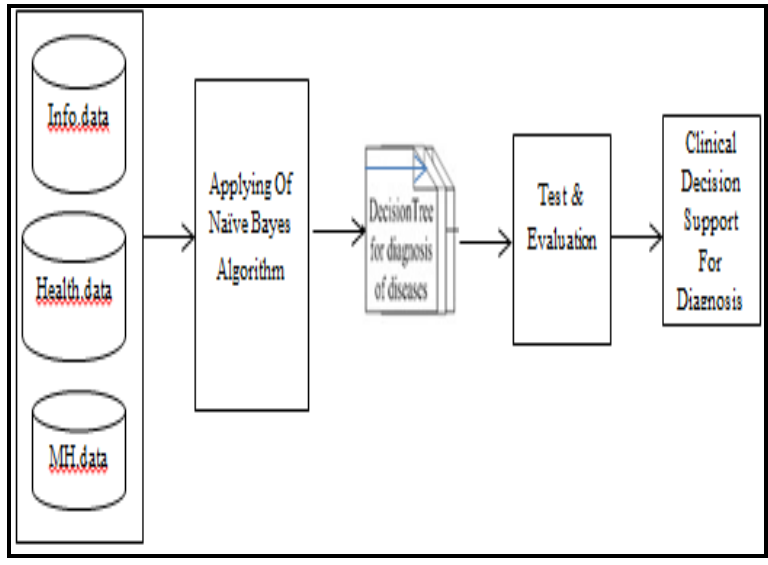

Fig 22 Steps Of Diagnostic

Thus, for variable's specialty above, we were profit from female information only, so according to table in Figure(23) above,(16\%) are diagnostic medically without Osteoporosis and $(84 \%)$ are with Osteoporosis.

In figure (23) show the prior probability that calculated according to equations in (1), with notation that all data of risk factors were discreet unless the data of ( age, weight , length) were continuous. 


\begin{tabular}{|c|c|c|c|c|c|c|c|}
\hline \multicolumn{8}{|c|}{ For Discreet Variable } \\
\hline $\mathrm{P}(\mathrm{X} 4=\mathrm{Yes} \mathrm{N} \mathbf{N})$ & & 0.71 & & $\mathrm{P}(\mathrm{X} 4=\mathrm{N} 0 \mathrm{~N} 0)$ & \multicolumn{3}{|c|}{0.29} \\
\hline $\mathrm{P}(\mathrm{X} 4=$ Yes Yes $)$ & & 0.12 & & $\mathrm{P}(\mathrm{X} 4=\mathrm{No} 0 \mathrm{Yes})$ & \multicolumn{3}{|c|}{0.88} \\
\hline $\mathrm{P}(\mathrm{X} 5=\mathrm{Yes}-\mathrm{N} 0)$ & & 0.26 & & $\mathrm{P}\left(\mathrm{X} 5=\mathrm{N}_{0} \mathrm{~N}_{0}\right)$ & \multicolumn{3}{|c|}{0.74} \\
\hline $\mathrm{P}(\mathrm{X} 5=$ =esYYes) & & 0.76 & & $\mathrm{P}(\mathrm{X} 5=\mathrm{N} 0$ Yes $)$ & \multicolumn{3}{|c|}{0.24} \\
\hline $\mathrm{P}(\mathrm{X} 6=\mathrm{Yes}[\mathrm{N} 0)$ & & 0.06 & & $\mathrm{P}(\mathrm{X} 6=\mathrm{N} 0 \mathrm{~N} 0)$ & \multicolumn{3}{|c|}{0.94} \\
\hline $\mathrm{P}(\mathrm{X} 6=\mathrm{Yes} Y \mathrm{Yes})$ & & 0.97 & & $\mathrm{P}(\mathrm{X} 6=\mathrm{N} 0$ Yes $)$ & \multicolumn{3}{|c|}{0.03} \\
\hline $\mathrm{P}(\mathrm{X}\rangle=\mathrm{Yes} \mathbf{N} \mathbf{0})$ & & 0.24 & & $\mathrm{P}\left(\mathrm{X} 7=\mathrm{N}_{0} \mathrm{~N}_{0}\right)$ & \multicolumn{3}{|c|}{0.97} \\
\hline $\mathrm{P}(\mathrm{X}\rangle=\mathrm{Yes} Y \mathrm{Yes})$ & & 0.76 & & $\mathrm{P}(X 7=\mathrm{N} 0$ Yes $)$ & \multicolumn{3}{|c|}{0.03} \\
\hline$\overline{P(X 8=Y e s(N 0)}$ & & 0.16 & & $\mathrm{P}\left(\mathrm{X} 8=\mathrm{N} 0 \mathrm{~N}_{0}\right)$ & \multicolumn{3}{|c|}{0.84} \\
\hline $\mathrm{P}(\mathrm{X} 8=$ =es Yes) & & 0.67 & & $\mathrm{P}(\mathrm{X} 8=\mathrm{No} 0 \mathrm{Yes})$ & \multicolumn{3}{|c|}{0.33} \\
\hline $\mathrm{P}(\mathrm{X} \mathrm{\theta}=\mathrm{Yes} \mathrm{N} \mathbf{\mathrm { N }})$ & & 0.03 & & $\mathrm{P}\left(\mathrm{X} \theta=\mathrm{N} 0 \mathrm{~N}_{0}\right)$ & \multicolumn{3}{|c|}{0.97} \\
\hline $\mathrm{P}(\mathrm{X} \theta=$ Yes Yes $)$ & & 0.46 & & $\mathrm{P}(\mathrm{X} \theta=\mathrm{No}$ |Yes $)$ & \multicolumn{3}{|c|}{0.44} \\
\hline \multicolumn{8}{|c|}{ For Continuous Variable } \\
\hline \multicolumn{4}{|c|}{ If class Yes } & \multicolumn{4}{|c|}{ If class $\mathrm{N}_{0}$} \\
\hline $\operatorname{Mean}(\mathrm{X} 1)$ & 63 & Variance $(\mathrm{X} 1)$ & 10 & $\operatorname{Mean}(X 1)$ & 48 & Variance $(\mathrm{X} \mathbf{1})$ & 8 \\
\hline $\operatorname{Mean}(\mathrm{X} 2)$ & 70 & \begin{tabular}{|l|} 
Variance $(\mathrm{X} 2)$ \\
\end{tabular} & 12 & $\operatorname{Mean}(\mathrm{X} 2)$ & 78 & Variance $\left(\mathrm{X}_{2}\right)$ & 8 \\
\hline $\operatorname{Mean}(X 3)$ & 152 & \begin{tabular}{|l|} 
Variance $(\mathrm{X} 3$ \\
\end{tabular} & 6 & $\operatorname{Mean}(X 3)$ & 155 & Variance $[X 3]$ & 5 \\
\hline
\end{tabular}

Fig 23: Form of The prior probability

Currently, we compute the posterior probability $\mathrm{P}(\mathrm{X} \mid \mathrm{Yes})$ and $\mathrm{P}(\mathrm{X} \mid \mathrm{No})$ by using equation (2).The Bayes Error Rate was compute as in equation (4).Figure(24) show the Osteoporosis Naïve Bayes classification, for each patent. Classification patents was compute by compare between $\mathrm{P}(\mathrm{X} \mid \mathrm{Yes})$ and $\mathrm{P}(\mathrm{X} \mid \mathrm{No})$ :

\section{IF $(P(X \mid$ Yes $)>P(X \mid N o), T H E N$, "PATENT WITH DESES",} ELSE, "PATENT WITHOUT DISES"

We have $(7 \%)$ cases are not parallel with their medical diagnose, that is because the problem of their bones are at first stage :(Osteopenia) yet.

\begin{tabular}{|c|c|c|c|c|c|c|c|}
\hline Ind $x$. & X5 & $\mathrm{X}_{6}$ & $\mathrm{X} 7$ & $\mathrm{x} 8$ & $\mathrm{x} 9$ & \begin{tabular}{|l|l|l|}
$\mathrm{P}(\mathrm{X} \mid \mathrm{NO})$ & $\mathrm{P}(\mathrm{X} Y \mathrm{YES})$ & Classification \\
\end{tabular} & dental Diagnosis \\
\hline 18 & 1 & 0 & 1 & 0 & 0 & 1 4.437E-09.34117E-05 WITH DISEAS & Osteoporosis \\
\hline 19 & 0 & 0 & 1 & 0 & 1 & $12.6 \mathrm{E}-08.83444 \mathrm{E}-06 \mathrm{WITH}$ DISEAS & Osteoporosis \\
\hline 20 & 0 & 1 & 0 & 0 & 1 & 0 0.0004295. 0000115973 WTTHOUT DIS & SOsteopentia \\
\hline 21 & 0 & 1 & 1 & 1 & 0 & 1 7.753E-08.000321155 WITH DISEAS & Osteoporosis \\
\hline 22 & 0 & 1 & 1 & 1 & 1 & 0 5.252E-06.006291113 WITH DISEAS & Osteopenia \\
\hline 23 & 0 & 1 & 1 & 1 & 0 & 0 9.797E-07.049856457 WITH DISEAS & Osteopenia \\
\hline 24 & 0 & 1 & 0 & 1 & 1 & 0 2.259E-05.006325107 WITH DISEAS & Osteopenia \\
\hline 25 & 0 & 0 & 0 & 1 & 0 & 0 7.883E-05.000612774 WTTHOUT DIS & SOSteopenia \\
\hline 26 & 0 & 0 & 0 & 1 & 1 & $00.0003665 .88169 \mathrm{E}-05$ WITH DISEAS & Osteopenia \\
\hline 27 & 0 & 1 & 1 & 1 & 0 & 0 1.642E-06.000665427 WITH DISEAS & Osteopenia \\
\hline 28 & 0 & 1 & 0 & 1 & 1 & 0 1.189E-05.009984603 WITH DISEAS & Osteopenia \\
\hline 29 & 0 & 0 & 1 & 0 & 1 & 00.0006873 .000867857 WITH DISEAS & Osteopenia \\
\hline 30 & 0 & 1 & 1 & 1 & 1 & 1 4.109E-08.004987292 WITH DISEAS & Osteopenia \\
\hline 31 & 0 & 0 & 1 & 1 & 1 & 1 1.206E-10 0.02195386 WITH DISEAS & Osteoporosis \\
\hline 32 & 0 & 0 & 0 & 1 & 1 & 1 1.275E-07.000220115 WITH DISEAS & Osteoporosis \\
\hline 33 & 0 & 0 & 1 & 1 & 1 & 0 2.013E-08 .89333E-05 WITH DISEAS & Osteopenia \\
\hline 34 & 0 & 1 & 1 & 1 & 0 & $\begin{array}{ll}0 & 6.52 \mathrm{E}-08.000294963 \text { WITH DISEAS }\end{array}$ & Osteopenia \\
\hline 35 & 0 & 1 & 1 & 1 & 1 & $17.355 \mathrm{E}-08.001736567$ WITH DISEAS & Osteoporosis \\
\hline 36 & 0 & 1 & 1 & 1 & 1 & $19.292 \mathrm{E}-120.02746024$ WITH DISEAS & Osteoporosis \\
\hline 37] & 0 & 1 & 0 & 1 & 0 & 1 1.777E-05.000685765 WITH DISEAS & Osteoporosis \\
\hline 38 & 0 & 0 & 1 & 1 & 1 & 0 1.003E-06.001160307 WTTH DISEAS & Osteopenia \\
\hline 39 & 0 & 1 & 1 & 1 & 1 & 1 1.336E-07.003507198 WITH DISEAS & Osteoporosis \\
\hline 40 & 0 & 1 & 1 & 0 & 1 & 0 6.451E-05.049265808 WTH DISEAS & Osteopenia \\
\hline 41 & 0 & 1 & 1 & 1 & 1 & $13.294 \mathrm{E}-12.009593803$ WITH DISEAS & Osteoporosis \\
\hline 42 & 1 & 0 & 1 & 0 & 1 & 0 9.222E-05.000957605 WTTHOUT D & Osteopenia \\
\hline
\end{tabular}

Fig 24:Form of Osteoporosis Naïve Bayes Classification for each patient

\section{RESULTS AND RECOMMENDATIONS} 7.1 Results:

1-By Computer Osteoporosis System - with different Data Bases tools-which designed in this paper, the problem of documenting the patient data was resolved, It was shown that implementing Bases as: FTP, ADO, URL, HTTP , made the documentation of information and their use in the following clinical check more easy and trusty. So Osteoporosis data base may enable to:
- Documenting all data related to disease for a study sample that may enable the treating physician to recall and update information simultaneously during visits.

- Insert new variables related to disease according to specialists in medicine or health staff.

- Help medical staff to take decisions related to diagnosis or expectations, especially system windows have been designed easy - use.

- Time and money saving as well as physical efforts.

2- HTTP have been uploaded (HTML) to work on FRAXWHO Fracture Risk Assessment Tool) and calculate the value of FRAX for each references then inserted to personal file of data base.

3- FTP has been used as internet protocol to transfer the personal file as a result of DXA in X-ray room and diagnosis to physician room.

4 -Techniques of Data Mining contributed to explore the patients behavior infected with the disease which helps to make appropriate decisions for the implementation of health action in suitable time which prevent (or reduce) the disease.

5- Decision tree has been built for osteoporosis cause after specifying most variables of the study.

6- According to the above-mentioned tree, rules have been reduced that may contribute in osteoporosis.

7- By calculating the two criterions: Accuracy (67\%) and Coverage $(82 \%)$, a simple but important rule was allocated: "IF the patient have a BMD<=-1SDT_Score and his risk factor type is (out of power) then he suffer from Hormone Problems".

8- The conditional probability of discrete variables has been estimated - ex. smoking (yes/no)- as a ratio (part-whole) mean, while the conditional probability of continues variables - ex. Age - according to function (2-8).

9- Using the Naive Bayes Classifier made it possible to trace the patients possible Osteoporosis successfully.

10- Results showed that (26) of (370) observation were classified incorrectly, so they were classified as unaffected, where they are diagnosed medically primarily (Osteopenia).

11- The error rate in the classification was not more than 0.07 .

12- The results can be summarized medically as in the following, they are considered risk factors of disease:

- If the patient has a BMD<=-1SDT_Score and his risk fac which leads to Deficiency of Hormone.

- If the patient's problem is Mineral Deficiency -which is in his power- then he has a Deficiency of Calcium \& Phosphor.

- A Deficiency of Hormone caused from either human body or drugs.

Finally the future scope of the idea is : Using comparison of Naïve Bayes and SVM classified for Early Deduction Of Osteoporosis

\section{REFERENCES}

[1] Peter C., Pablo H., Rolf S., Jaap V., Alessandro Z.,1997, Discovering Data Mining: From Concept to Implementation, Prentice Hall. 
[2] Melton L., Riggs B., Fracture Risk After Bilateral Oophorectomy in Elderly Women, J. of Bone and Mineral Research, Vol. 18, No. 5, 2003,900-905

[3] William C.Shiel Jr.,2015,Osteoporosis:Get facts or Diet, Treatment and Risk factor of causes, MedicineNet, Inc http://www.medicineNet.com/Osteoporosis/article.com

[4] Bone Mineral Density Testing: https://www.health.ny.gov/diseases/conditions/osteoporo sis/bone_density_testing.htm,2012

[5] Reiner B. , Bertha F.,2009, " Osteoporosis : Diagnosis, Prevention, Therapy ", 2nd ed., Springer,P72.

[6] American Bone Health, 2015, https://americanbonehealth.org/what-you-shouldknow/about-t-scores

[7] Peter Rob and Carlos Coronel, 2009,Database Systems: Design, Implementation, and Management, Eighth Edition, United States ,Thomas- Course Technology

[8] Sateesh, B., 2011, JAVA, C\#, VB.net, SQL, C, Computer Sci Free Computer Programming Tutorials. Travel, P. 2007 Modeling and Simulation Design. AK Peters Ltd.

[9] Hamse Y Mussa, John BO Mitchell and Robert C Glen,2013, Full "Laplacianised" posterior naive Bayesian algorithm, Journal of Cheam informatics

[10] Dean, and Tamara, 2010, Network+ Guide to Networks, Delmar, , Pp.168-171

[11] Bradley,Mitchell,2015, FTP - What Does FTP Stand For, About.com

[12] File Transfer Protocol (FTP) Instructions For Submitting Reports to SCO,2014, California State Controller's Office http://www.sco.ca.gov/Files-ARDLocal/LocRep/ftp_Reporting_Instructions.pdf

[13] Peter R. Egli,2015, FTP FILE TRANSFER PROTOCOL, INTRODUCTION TO FTP, THE INTERNET'S STANDARD FILE TRANSFER PROTOCOL

[14] Kozierok, Charles M. ,2005, The TCP/IP Guide v3.0, Retrieved From, P10 http://www.tcpipguide.com/free/t_FTPOverviewHistorya ndStandards.htm

[15] Bradley, Mitchell,2015,HTTP,About.com

[16] Ruellan, H. and Peon R.,2013, HPACK - Header Compression for HTTP/2.0, Internet-Draft draft-ietfhttpbis-header-compression-05.

[17] Barry de Miner, 2006, Decision Trees for Business Intelligence and Data Mining: Using SAS, SAS Institute Inc., Cary, NC, USA,p60

[18] FÜrnkranz, J, " Separate-and-conquer rule learning", Artificial Intelligence Review ,Vol.13(1),1999,Pp. 3-54.

[19] Stefanowski J., 2001,Algorithms of Decision Rule Induction in Data Mining, Poznan ,Poland,P34.

[20] Lior R, Oded M.,2008, Data Mining With decision Trees : Theory and Applications, World Scientific Publishing Co. Pte. Ltd,pp133-143.

[21] Donna Giri U., Rajendra A., Roshan J. , Vinitha S. , Teikcheng L. ,Thajudin A. , Jasjit S., " Automated diagnosis of Coronary Artery Disease affected patients using LDA, PCA,ICA and Discrete Wavelet Transform", Knowledge-Based Systems, Vol. 37,2013,Pp(274-282)

[22] Oded M., Lior R., 2010, Data Mining and Knowledge Discovery Handbook, $2^{\text {nd }}$ edition, Springer, Pp.621-622

[23] Xindong ,W. Vipin K., 2009, Data Mining and Knowledge Discovery Series, Chapman \& Hall,P163.

[24] Luis T., 2011,Data Mining with R, Learning with Case Studies ,Chapman \& Hall,P211.

[25] Stephan T., 2011, Data Mining and Statistics for Decision Making, 1st ed., John Wiley \& Sons.,P492.

[26] Banks D. , House L. ,McMorris F. , Arabie P., Gaul W., 2004, Classification, Clustering, and Data Mining Applications, Springer,P487.

[27] Trevor H., Robert T. , Jerome F., 2008,The Elements of Statistical Learning :Data Mining, Inference and Prediction ,2nd ed., Springer,P21-211.

[28] Fukunaga, K., 1990,Introduction to Statistical Pattern Recognition ,2nded ,Academic Press,P196.

[29] Devijver P., Kittler J.,1982,Pattern Recognition: A Statistical Approach, Prentice-Hall,P56.

[30] Liangxiao J., Dianhong W., Zhihua C., 2007,Advanced Intelligent Computing Theories and Applications. With Aspects of Artificial Intelligence, Lecture Notes in Computer Science, pp 475-484

[31] Irina R., "An empirical study of the naive Bayes classifier", IJCAI 2001 Workshop on Empirical Methods in Artificial.

[32] A. Silberschatz, H.F. Korth, S. Sudarshan,2010, Database Systems Concepts, 6th Edition, McGraw-Hill.

[33] Narayan,S. Umanath \& Richard W. Scamell, 2015,Data Modeling \& Data Design,2nd Edition, Cengage Learning.

\section{APPENDESIS \\ APPENDIX (1)}

\section{Appendix(1-A) rule Induction Form1}

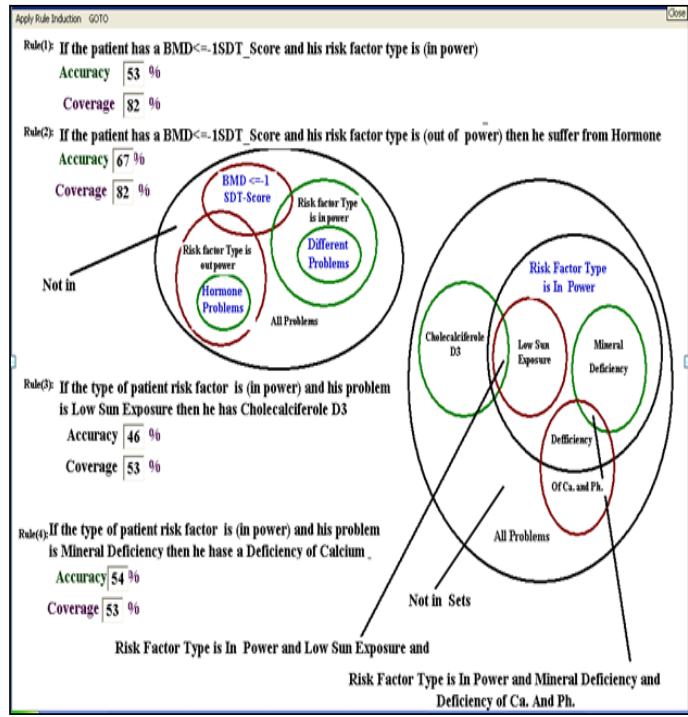

Appendix(1-B) rule Induction Form2 


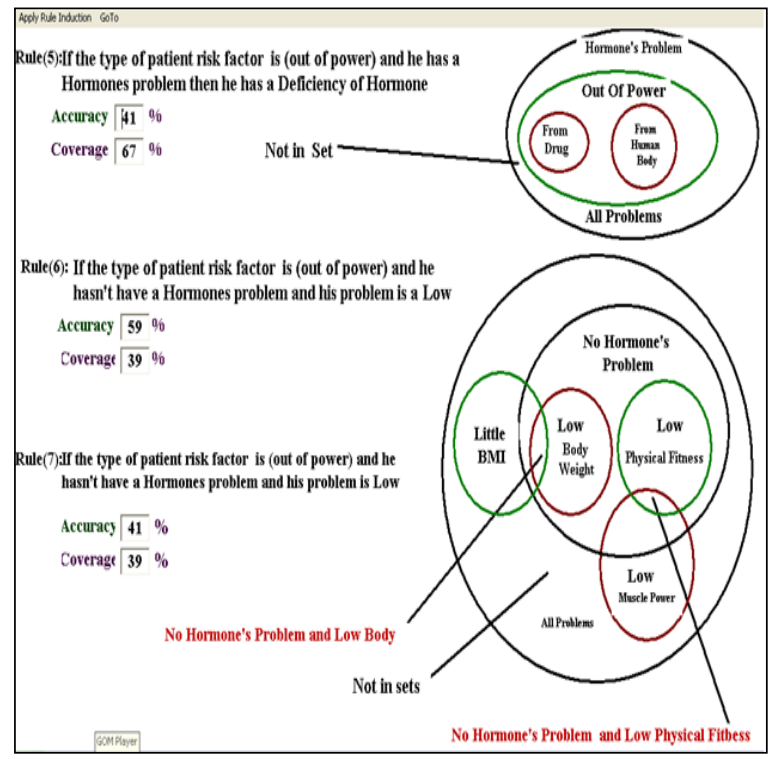

\section{APPENDIX(2):}

Appendix(2-A):Naïve Bayes Tree Form1

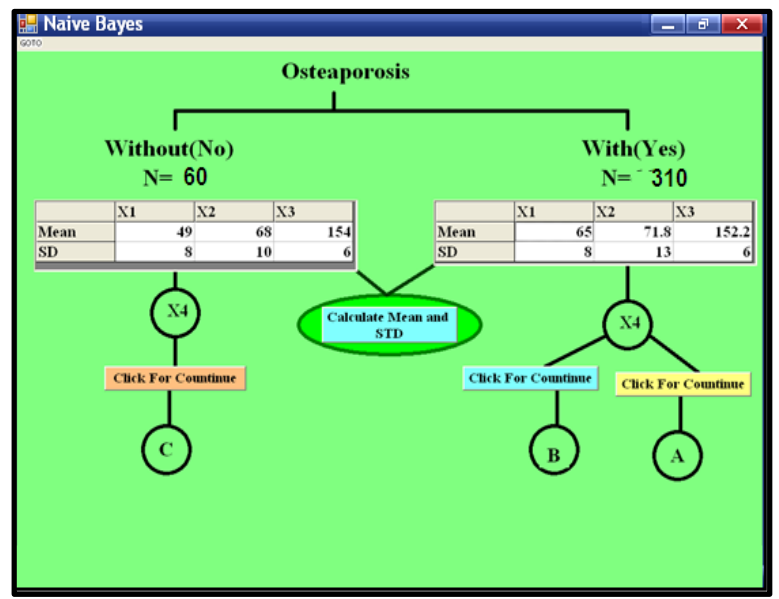

Appendix(2-B):Naïve Bayes Tree Form2

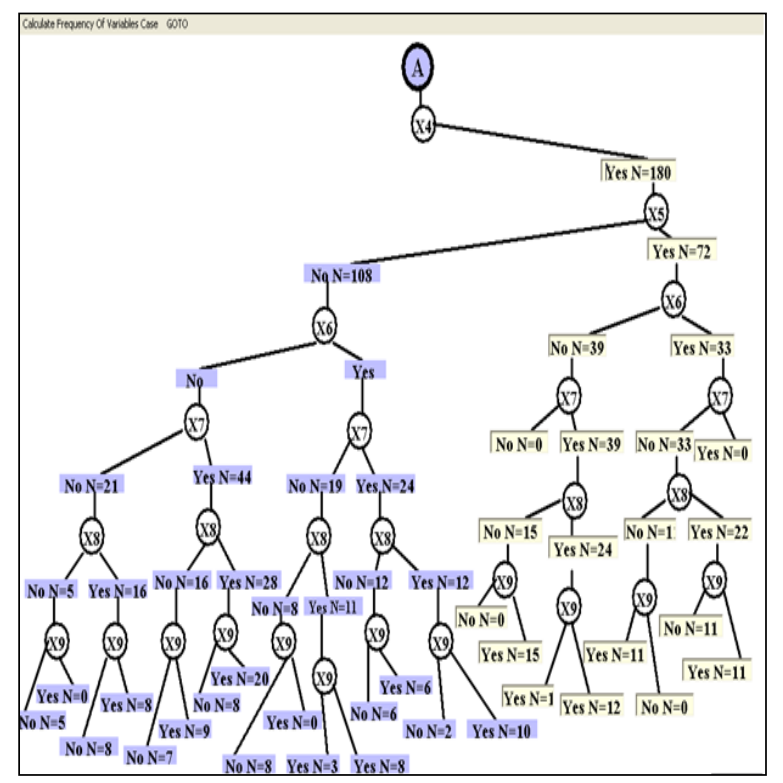

Appendix(2-C):Naïve Bayes Tree Form3

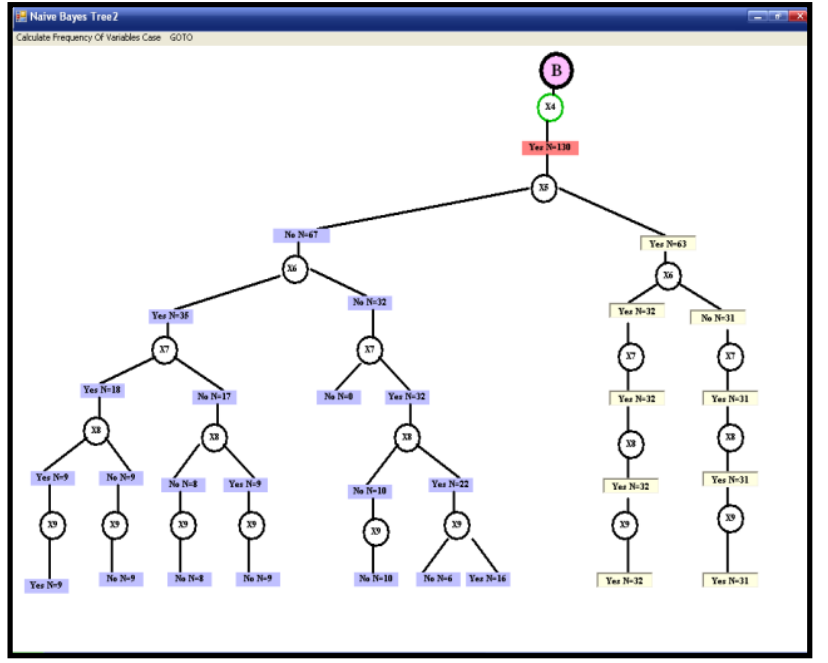

Appendix(2-D):Naïve Bayes Tree Form 4

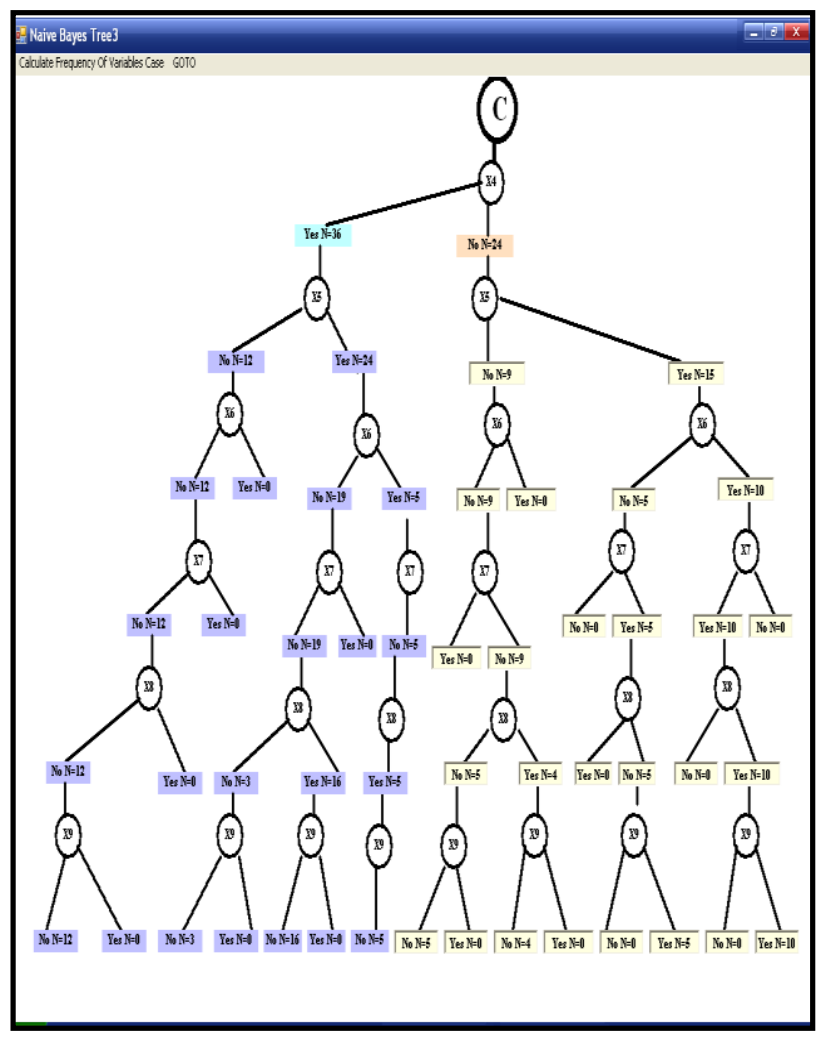

